\title{
Absorption Line Surveys at Intermediate Redshift
}

\author{
Sébastien Muller \\ Onsala Space Observatory, Sweden \\ email: mullers@chalmers.se
}

\begin{abstract}
The $\mathrm{z}=0.89$ spiral galaxy located on the line of sight to the quasar PKS1830-211 is right now the extragalactic object with the largest number of detected molecular species (34, plus isotopic variants). This remarkable molecular inventory was recently built after an unbiased spectral survey of molecular absorption lines with the Australia Telescope Compact Array in the $7 \mathrm{~mm}$ band. We present the results of this survey, as a showcase of the various interests of molecular absorption studies at intermediate redshift: chemical inventory and gas properties in distant galaxies, chemical enrichment history of the Universe, measurements of the CMB temperature as a function of $\mathrm{z}$, and constraints on the constancy of fundamental constants.
\end{abstract}

Keywords. quasars: absorption lines, quasars: individual (PKS1830-211), radio lines: galaxies, galaxies: ISM, ISM: molecules, surveys, cosmic microwave background.

\section{Introduction}

Interstellar molecules were first detected in absorption along the line of sight toward bright nearby stars. Now, we know that the interstellar medium (ISM) is home of more than 150 molecular species and astrochemistry has been a rapidly growing field. Moreover, recent surveys have open the door of extragalactic astrochemistry (e.g., Wang et al. (2004), Martín et al. (2006), Martín et al. (2011), Costagliola et al. (2011)), although only the central region of the bright and nearby sources reveal their molecular richness.

After the discovery of molecular absorption in five objects located at intermediate redshifts $(0.24<\mathrm{z}<0.89$, cf a review by Combes et al.2008), it is now possible to explore the chemical content in the disk of galaxies up to significant redshifts. The interests of absorption studies for distant objects are manifold:

- There is no distance dilution, absorption can be detected as easily at high redshift as in the Local Universe. The only limitation is the need for background continuum.

- It offers outstanding spatial resolution, at the apparent angular size of the continuum source. For example at mm wavelengths, the continuum beam pencil through the $\mathrm{z}=0.89$ galaxy located in front of PKS1830-211 is of the order of the parsec scale, projected in the plane of this galaxy.

- Excitation is simple in low density $\left(\sim 100-1000 \mathrm{~cm}^{-3}\right)$ gas, where collisions are inefficient and the excitation is coupled with the radiative field. In the absence of other local heating source, the excitation is dominated by CMB photons, so that rotation temperatures nearly equal the CMB temperature.

- Optical depths can be obtained directly from the line-to-continuum flux density ratio, and column densities can be derived straightforwardly, as a consequence of the two previous points. This is not the case in extragalactic emission studies, limited by the beam resolution. 
- Deep inventory of the molecular content can be obtained, including species that would be nearly impossible to detect in emission due to distance dilution.

- High-z molecular absorbers are valuable cosmological probes (see $\S 5$ hereafter).

- Transitions difficult to observe from the ground, due to poor atmospheric transitions or challenging techniques, may be redshifted to a more accessible frequency window. For example, submillimeter lines of light hydrides (cf. the wealth of Herschel results presented in this symposium) could be studied in absorption, at redshifted frequency, with ALMA.

\section{Extragalactic radio-mm molecular absorbers}

The number of extragalactic radio-mm molecular absorbers known is very limited, and only five have been discovered to date at intermediate redshifts $(0.24<\mathrm{z}<0.89$, cf a review by Combes (2008)), despite active searches (e.g., Wiklind \& Combes (1996a), Drinkwater et al. (1996), Murphy et al. (2003), Curran et al.(2004, 2006, 2008)). They are listed in Table 1, together with some other nearby extragalactic absorbers. We do not include here the $\mathrm{H}_{2} / \mathrm{CO}$ absorbers detected from optical/UV spectroscopy, such as those discussed by Noterdaeme et al.(2011), since a deep exploration of their molecular content remains currently out of reach.

For the majority of absorbers, the absorption occurs in the host-galaxy of the continuum source. In the case of the last three entries in Table 1, the absorbers correspond to intervening galaxies physically unrelated to the quasar. As molecular gas is mostly concentrated within the inner disk of spiral galaxies, it is not surprising that their background quasars are strongly gravitationally lensed, as a result of the small impact parameter.

The most notable $\mathrm{mm}$ molecular absorber at intermediate redshift is the $\mathrm{z}=0.89$ spiral galaxy toward the quasar PKS1830-211. It has the highest redshift, the brightest millimeter continuum and the largest amount of absorbing material. The intervening galaxy appears as a face-on spiral (Winn et al. (2002); Fig.1 left) and acts as a gravitational lens, splitting the background quasar into two main compact cores (NE and SW), separated by 1" and embedded in a fainter Einstein ring, seen at $\mathrm{cm}$ wavelengths (Jauncey et al. (1991)). Molecular absorption is seen toward both compact images (Wiklind \& Combes (1998), Muller et al. (2006)), separated in velocity by $\sim 150 \mathrm{~km} \mathrm{~s}^{-1}$ (Fig.1 right). The SW and NE images of the quasar fall on either side of the $z=0.89$ galaxy bulge at galactocentric radii of $\sim 2$ and $\sim 4 \mathrm{kpc}$, respectively.

The SW line of sight is the most molecular rich, with a $\mathrm{H}_{2}$ column density of $\sim 2 \times 10^{22} \mathrm{~cm}^{-2}$, while the NE line of sight is only detected in a handful of molecular species $\left(\mathrm{N}\left(\mathrm{H}_{2}\right) \sim 10^{21} \mathrm{~cm}^{-2}\right)$. These $\mathrm{H}_{2}$ column density estimates are consistent with X-ray absorption measurements (Mathur \& Nair (1997)), the differential $\mathrm{A}_{V}$ extinction between the NE and SW quasar images (Falco et al. (1999)) and the scaling from $\mathrm{C}_{2} \mathrm{H}$ column densities (Muller et al. (2011)) using a Galactic conversion factor (Lucas \& Liszt (2000), Gérin et al. (2010)). The density is of the order of $2000 \mathrm{~cm}^{-3}$ toward the SW absorption (Henkel et al. (2009)).

\section{The ATCA $7 \mathrm{~mm}$ spectral survey toward PKS1830-211}

With the Australian Telescope Compact Array (ATCA), we have recently conducted the first unbiased spectral survey toward PKS1830-211 (cf. Muller et al. (2011)), covering the frequency range $30-50 \mathrm{GHz}$, which corresponds to the rest-frame interval $57-94 \mathrm{GHz}$ at $\mathrm{z}=0.89$. Such a survey has been made possible by the new broadband backend system (CABB) on ATCA, providing an instantaneous bandwidth of $4 \mathrm{GHz}$ and $1 \mathrm{MHz}$ spectral 
Table 1. Extragalactic radio-mm molecular absorbers.

\begin{tabular}{|c|c|c|c|c|c|}
\hline Source & $z_{a b s}$ & $\begin{array}{l}\text { Continuum } \\
\text { flux density } \\
@ 3 \mathrm{~mm}(\mathrm{Jy})^{a}\end{array}$ & $\begin{array}{c}\mathrm{N}\left(\mathrm{H}_{2}\right) \\
\left(10^{20} \mathrm{~cm}^{-2}\right)\end{array}$ & Molecules detected & Ref. \\
\hline \multicolumn{6}{|c|}{ Absorption in the host-galaxy of the continuum source } \\
\hline Cen A & 0.002 & 6 & 2 & $\begin{array}{c}\mathrm{CO}, \mathrm{OH}, \mathrm{NH}_{3}, \mathrm{CN}, \mathrm{HCO}^{+}, \mathrm{HCN}, \mathrm{N}_{2} \mathrm{H}^{+} \\
\mathrm{CS}, \mathrm{H}_{2} \mathrm{CO}, \mathrm{c}-\mathrm{C}_{3} \mathrm{H}_{2}\end{array}$ & $(1)$ \\
\hline NGC1052 & 0.005 & 0.6 & 25 & $\mathrm{OH}, \mathrm{CO}, \mathrm{HCO}^{+}, \mathrm{HCN}$ & $(2)$ \\
\hline $3 \mathrm{C} 293$ & 0.045 & 0.19 & 0.2 & $\mathrm{CO}, \mathrm{HCO}^{+}, \mathrm{HCN}$ & $(3)$ \\
\hline $4 \mathrm{C} 31.04$ & 0.060 & 0.16 & 0.1 & $\mathrm{CO}, \mathrm{HCO}^{+}, \mathrm{HCN}$ & $(4)$ \\
\hline PKS $1413+135$ & 0.247 & 0.5 & 5 & $\mathrm{CO}, \mathrm{CN}, \mathrm{HCO}^{+}, \mathrm{HCN}, \mathrm{HNC}$ & $(5)$ \\
\hline B $1504+377$ & 0.673 & 0.4 & 5 & $\mathrm{CO}, \mathrm{HCO}^{+}, \mathrm{HCN}, \mathrm{HNC}$ & $(6)$ \\
\hline \multicolumn{6}{|c|}{ Absorption in an intervening galaxy } \\
\hline B $0218+357$ & 0.685 & $0.5^{b}$ & 40 & $\begin{array}{c}\mathrm{CO}, \mathrm{NH}_{3}, \mathrm{H}_{2} \mathrm{O}, \mathrm{HCO}^{+}, \mathrm{HCN}, \mathrm{HNC} \\
\mathrm{H}_{2} \mathrm{CO}, \mathrm{CS}, \mathrm{H}_{2} \mathrm{~S}\end{array}$ & (7) \\
\hline PKS 0132-097 & 0.765 & 0.4 & - & $\mathrm{OH}$ (only, so far) & (8) \\
\hline PKS 1830-211 & 0.886 & $3^{b}$ & $200 / 10^{c}$ & $\begin{array}{c}\text { now } 34 \text { species (not including isotopic variants), } \\
\text { see Table } 2\end{array}$ & (9) \\
\hline
\end{tabular}

\section{Notes:}

${ }^{a}$ most of the sources have time variable flux.

${ }^{b}$ gravitationally lensed. This is the total flux density.

${ }^{c}$ toward the SW and NE images, respectively.

References:

(1): Gardner \& Whiteoak (1976), Seaquist \& Bell (1986), Seaquist \& Bell (1990), Israel et al. (1990), Eckart et al. (1990), Wiklind \& Combes (1997a), Muller \& Dinh-V-Trung (2009), Espada et al. (2010); (2): Omar et al. (2002), Liszt \& Lucas (2004); (3): Evans et al. (1999), García-Burillo et al. (in prep.); (4): GarcíaBurillo et al. (2007); (5): Wiklind \& Combes (1997b); (6): Wiklind \& Combes (1996a); (7): Wiklind \& Combes (1995), Menten \& Reid (1996), Henkel et al. (2005), Muller et al. (2007), Kanekar (2011), Muller et al. (in prep.); (8): Kanekar et al. (2005); (9): Wiklind \& Combes (1996b), Gérin et al. (1997), Wiklind \& Combes (1998), Chengalur et al. (1999), Menten et al. (1999), Muller et al. (2006), Henkel et al. (2008), Menten et al. (2008), Bottinelli et al. (2009), Henkel et al. (2009), Muller et al. (2011).

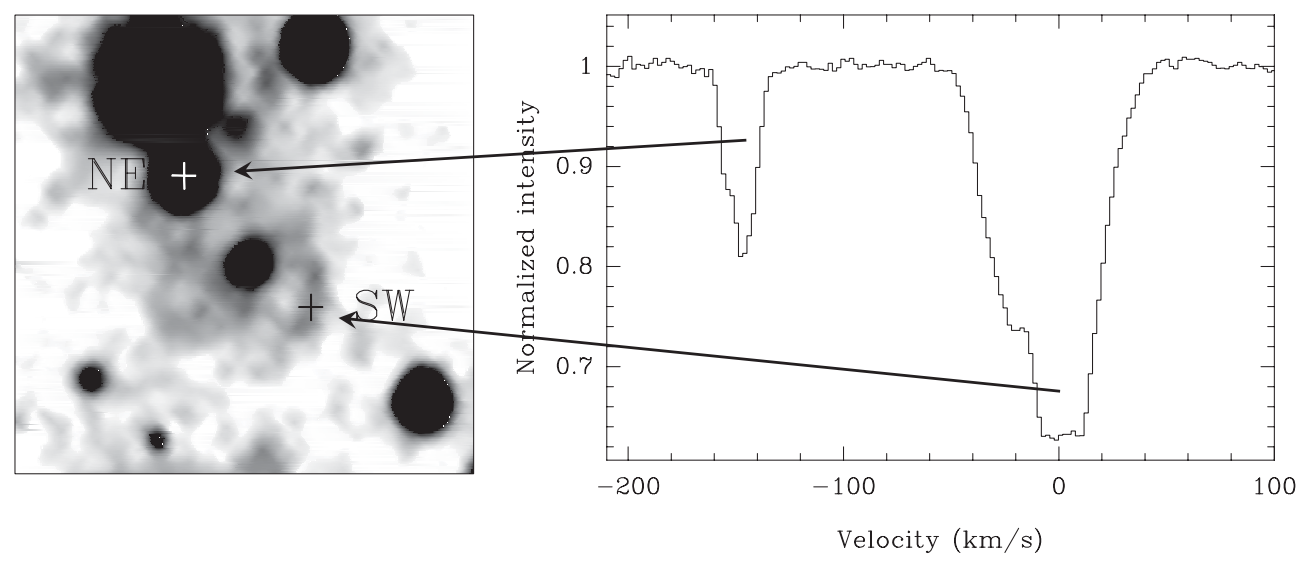

Figure 1. Left: Hubble Space Telescope image of the $\mathrm{z}=0.89$ face-on spiral galaxy toward the quasar PKS1830-211. The positions of the NE and SW lensed images of the background quasar, separated by 1", are indicated by the crosses. Note the very high extinction along the SW line of sight, where the image of the quasar is invisible in this optical image. Right: Spectrum of the $\mathrm{HCO}^{+} 2-1$ line observed with the Plateau de Bure interferometer, showing the two velocity components toward the NE and SW images of the quasar. Adapted from Muller et al. (2006).

resolution. The whole survey was done in a total of 18 hours of telescope time, allowing us to achieve a resulting optical depth sensitivity of a few per mil.

There are only few lines with optical depth greater than 0.1 in the survey, as can be seen on Figure 2, where the cumulative number of detected lines is given as a function of the optical depth. The median opacity is $\sim 0.01$, given the noise level. In total, we 
have been able to identify 28 different molecular species (plus some isotopic variants). A family picture is shown in Figure 3. The line identification was rather easy (no U-lines), because the line density is low ( $\sim 4$ lines/ GHz on average over the whole $7 \mathrm{~mm}$ band), and, as the excitation is coupled with the $\mathrm{CMB}$, we could filter only low-energy transitions $\left(\mathrm{E}_{\text {low }}<20-30 \mathrm{~K}\right)$ during the search in molecular databases, thereby reducing heavily the list of line candidates. Here, we especially acknowledge and emphasize the convenience of on-line molecular databases, such as the Cologne Database for Molecular Spectroscopy (Müller et al. (2001)) and the Jet Propulsion Laboratory Molecular Spectroscopy (Pickett et al. (1998)), which we used intensively for line identification.

The molecular inventory, listed in Table 2, includes common molecules detected in the ISM, formed of the H, C, N, O, S and Si elements. There are ions (e.g., $\mathrm{HCO}^{+}, \mathrm{HOC}^{+}$, $\mathrm{N}_{2} \mathrm{H}^{+}, \mathrm{SO}^{+}$), highly reactive radicals (e.g., $\mathrm{C}_{2} \mathrm{H}, \mathrm{C}_{4} \mathrm{H}$ ), unsaturated carbon chains (e.g., l- $\left.\mathrm{C}_{3} \mathrm{H}, \mathrm{l}-\mathrm{C}_{3} \mathrm{H}_{2}, \mathrm{C}_{4} \mathrm{H}\right)$, saturated species $\left(\mathrm{CH}_{3} \mathrm{OH}, \mathrm{CH}_{3} \mathrm{NH}_{2}\right)$, all together demonstrating that a complex and active chemistry is at work in these molecular clouds located in the disk of a $z=0.89$ galaxy. Certainly, the molecular inventory in Table 2 is not complete, owing to the observational bias toward species with a high dipole moment, a low partition function and low energy transitions within the frequency coverage of the survey. Future observations will undoubtedly increase the list. Still, it is interesting to note that this $\mathrm{z}=0.89$ galaxy toward PKS1830-211 is right now the extragalactic source with the largest number of detected molecular species!

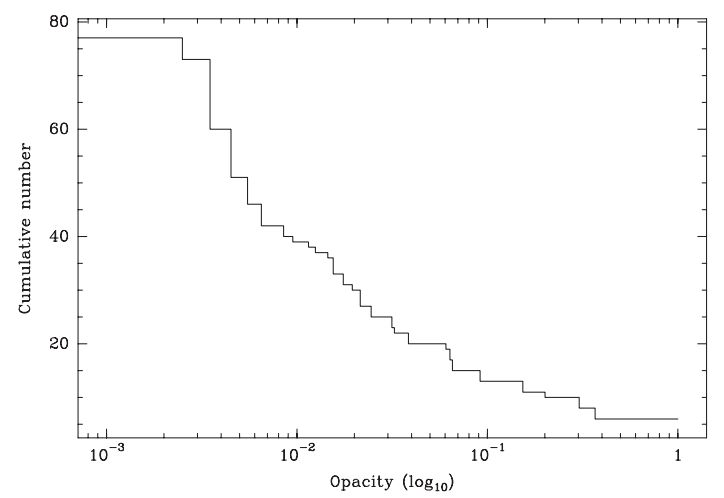

Figure 2. Cumulative number of lines detected in the ATCA $7 \mathrm{~mm}$ survey toward PKS1830-211, as a function of opacity. This illustrates the obvious needs for large instantaneous bandwidth and sensitivity for spectral survey.

Most of the lines are optically thin, so that the associated column densities can be derived directly and accurately (assuming that the rotation temperature $\mathrm{T}_{\text {rot }}=\mathrm{T}_{\mathrm{CMB}}=5.14 \mathrm{~K}$ at $\mathrm{z}=0.89$ ). Taking the $\mathrm{H}_{2}$ column densities given above, we can then obtain the fractional molecular abundances.

It is very tempting to compare these abundances with those observed in local sources, in order to characterize the chemistry. Such a comparison is shown in Figure 4, where we include the fractional abundances measured in diffuse clouds (Liszt et al. (2008) and references therein) in translucent clouds (Turner (2000)), in the dark cloud TMC 1 (Ohishi et al. (1992)), in the Galactic Center molecular cloud Sgr B2(N) (Nummelin et al. (2000)) in the the N113 region in the Large Magellanic Cloud (Wang et al. (2009)) and in the nuclear region of the nearby starburst galaxies NGC253 (Martín et al. (2006)) and NGC4945 (Wang et al. (2004)). There is a large scatter of up to nearly two orders of magnitude between the abundances in different sources, reflecting the different 
Table 2. Census of species detected at $\mathrm{z}=0.89$ toward PKS1830-211(SW).

\begin{tabular}{|c|c|c|c|c|c|c|}
\hline 1 atom & 2 atoms & 3 atoms & 4 atoms & 5 atoms & 6 atoms & 7 atoms \\
\hline $\begin{array}{l}(\mathrm{H})^{(d)} \\
(\mathrm{C})^{(i)}\end{array}$ & $\begin{array}{c}(\mathrm{OH})^{(d)} \\
(\mathrm{CO})^{(b c i)} \\
(\mathrm{CS})^{(a f)} \\
\mathrm{SiO}^{(j) \dagger} \\
\mathbf{N S} \\
\mathbf{S O} \\
\mathbf{S O}^{+}\end{array}$ & $\begin{array}{c}\left(\mathrm{H}_{2} \mathrm{O}\right)^{(h)} \\
\mathrm{C}_{2} \mathrm{H}^{(e)} \\
\mathrm{HCN}^{(a e f)} \diamond \\
\mathrm{HNC}^{(a e f)} \diamond \\
\mathrm{N}_{2} \mathrm{H}^{+(a)} \\
\mathrm{HCO}^{+}(\text {aef }) \\
\mathbf{H C O}^{-} \\
\mathbf{H O C}^{+} \\
\left(\mathrm{H}_{2} \mathrm{~S}^{(f)}\right. \\
\mathbf{C}_{2} \mathbf{S}\end{array}$ & $\begin{array}{c}\left(\mathrm{NH}_{3}\right)^{(g h)} \\
\mathrm{H}_{2} \mathrm{CO}{ }^{(c e)} \\
\underline{\mathbf{l}-\mathbf{C}_{3} \mathbf{H}} \\
\mathbf{H N C O} \\
\mathbf{H}_{2} \mathbf{C S}\end{array}$ & 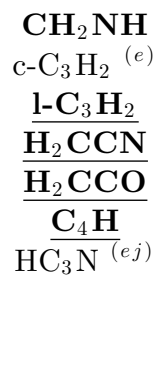 & $\begin{array}{l}\mathrm{CH}_{3} \mathrm{OH} \\
\mathrm{CH}_{3} \mathrm{CN}\end{array}$ & $\begin{array}{l}\frac{\mathrm{CH}_{3} \mathrm{NH}_{2}}{\mathrm{CH}_{3} \mathbf{C}_{2} \mathbf{H}} \\
\underline{\mathrm{CH}_{3} \mathrm{CHO}}\end{array}$ \\
\hline
\end{tabular}

Notes: In parenthesis, species detected in other studies; bold face, new detections; underlined: first extragalactic detections.

$\bigcirc$ including $\mathrm{H}^{13} \mathrm{CO}^{+}, \mathrm{HC}^{18} \mathrm{O}^{+}$and $\mathrm{HC}^{17} \mathrm{O}^{+} ; \triangle$ including $\mathrm{H}^{13} \mathrm{CN}$ and $\mathrm{HC}^{15} \mathrm{~N} ; \diamond$ including $\mathrm{HN}^{13} \mathrm{C}_{\text {and }}$ $\mathrm{H}^{15} \mathrm{NC} ; \dagger$ including ${ }^{29} \mathrm{SiO}$.

References: (a) ?; (b) Gérin et al. (1997); (c) Wiklind \& Combes (1998); (d) Chengalur et al. (1999); (e) Menten et al. (1999); (f) Muller et al. (2006), including isotopologues; (g) Henkel et al. (2008); (h) Menten et al. (2008); (i) Bottinelli et al. (2009); (j) Henkel et al. (2009).

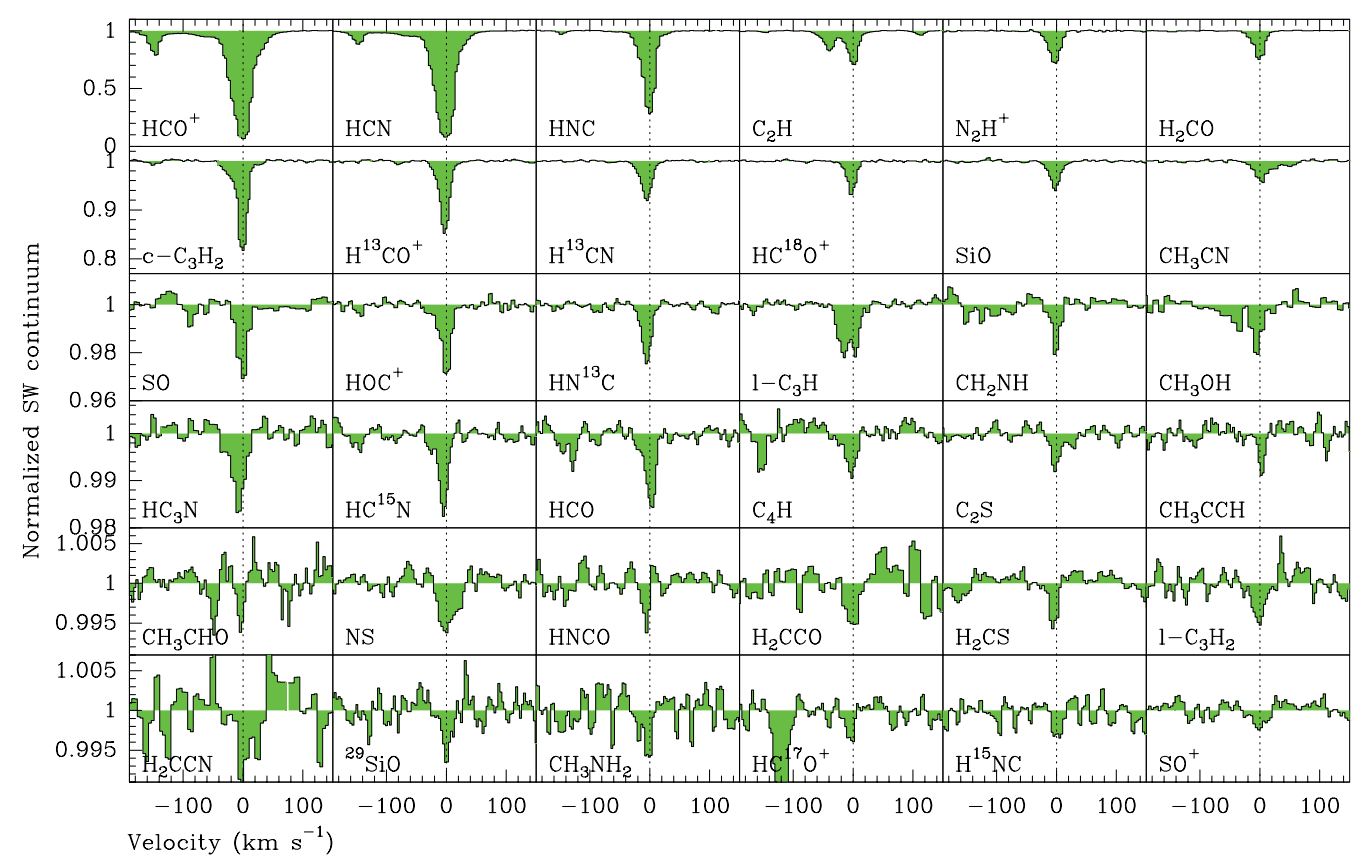

Figure 3. A family picture of molecular species detected in the ATCA $7 \mathrm{~mm}$ survey toward PKS1830-211. Note the change of absorption depth scale (top-left to bottom-right corner) from the deepest $(100 \%) \mathrm{HCO}^{+} / \mathrm{HCN}$ lines down to the faintest (a few per mil) lines $\left(\mathrm{HC}^{17} \mathrm{O}^{+} / \mathrm{H}^{15} \mathrm{NC} / \mathrm{SO}^{+}\right)$. The absorption toward the $\mathrm{NE}$ image of the quasar is seen at a velocity of $-150 \mathrm{~km} \mathrm{~s}^{-1}$ on the $\mathrm{HCO}^{+}$spectrum.

environment conditions and chemical setups. Here, the common sources of uncertainty have to be kept in mind: beam averaging (especially for emission lines in extragalactic sources), uncertainty on $\mathrm{H}_{2}$ column densities, excitation and opacity effects, as well as on the background continuum covering factor for absorption lines. For the latter, we assume 


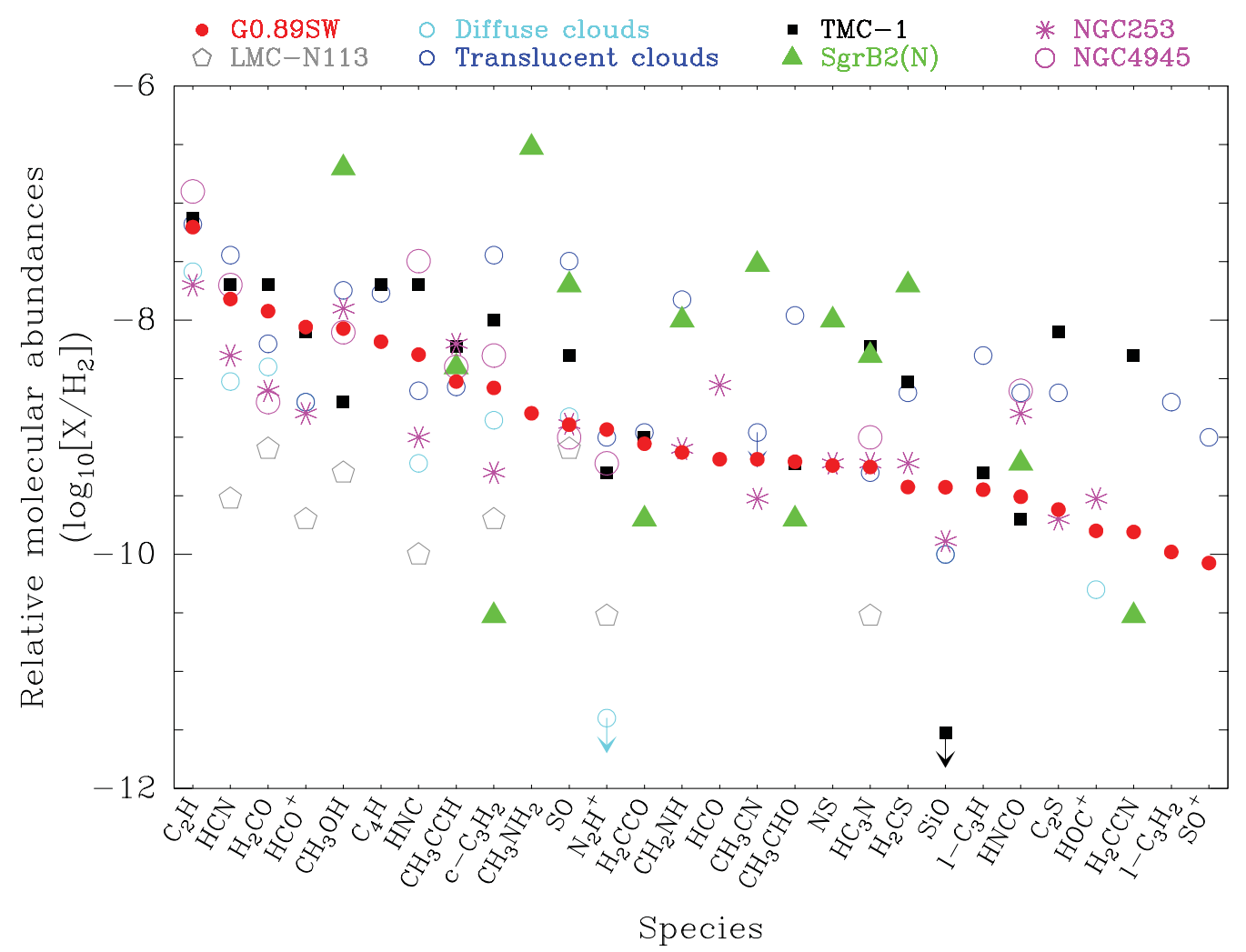

Figure 4. Comparison of fractional molecular abundances in the $\mathrm{z}=0.89$ galaxy (SW image) and that in various other objects. Species are ordered from the most to less abundant toward PKS1830-211(SW).

a source covering factor of unity for all species, so that the apparent optical depths and column densities are strictly speaking lower limits.

Overall, we find that the molecular abundances toward the SW image are intermediate between those in diffuse and translucent clouds, and clearly deviate from those observed toward the Galactic Center molecular cloud Sgr B2N. Toward the NE image, molecular abundances are close to those observed in diffuse clouds. Despite the look-back time of $\sim 7$ Gyr, the chemistry does not appear significantly different from that in Galactic clouds. It will be interesting to obtain additional information on the metallicity, elemental abundances and PAHs. We note that the $\mathrm{H}_{2} \mathrm{CO} / \mathrm{H}_{2} \mathrm{CS}$ ratio of $32 \pm 6$ is consistent with the present $\mathrm{O} / \mathrm{S}$ cosmic abundance ratio (33:1, Lodders (2003)). In contrast, isotopic ratios are markedly different from that in local sources (see $\S 5.2$ ).

\section{Time variations of the absorption profiles}

The monitoring of the $\mathrm{HCO}^{+} 2-1$ line over 13 years (1995-2008) has revealed the time variability of the absorption profile (Muller \& Guélin (2008)). The changes in absorption depths are shown in Figure 5. For example, one can see that the line at $\sim-150 \mathrm{~km} \mathrm{~s}^{-1}$ (arising toward the NE image) almost completely vanished in 2006, and reappeared in 2008. Changes in the NE line and wings of the SW line are correlated, indicating that a change in the morphology of the quasar is in cause. Past VLBA observations by Jin 
et al. (2003) have revealed variations in the distance between the NE and SW cores, of up to 200 micro-arcseconds within 8 months, corresponding to $1.6 \mathrm{pc}$ projected in the plane of the $\mathrm{z}=0.89$ galaxy. Those changes were interpreted as due to the recurrent ejection of plasmons along a helicoidal jet by Nair et al. (2005). As a consequence, the continuuum illumination is scanning a slightly changing line of sight through the disk of the intervening galaxy.

This configuration offers the unique chance to investigate the molecular clouds structure in the disk of a $z=0.89$ galaxy. Very-long-baseline mm-interferometry could help monitoring the continuum morphology and location of molecular absorption at regular time interval, yielding a measurement of their size. Alternatively, a high-sensitivity spectral monitoring of the absorption profile of various molecular species could probe their spatial correlation at sub-parsec scale.

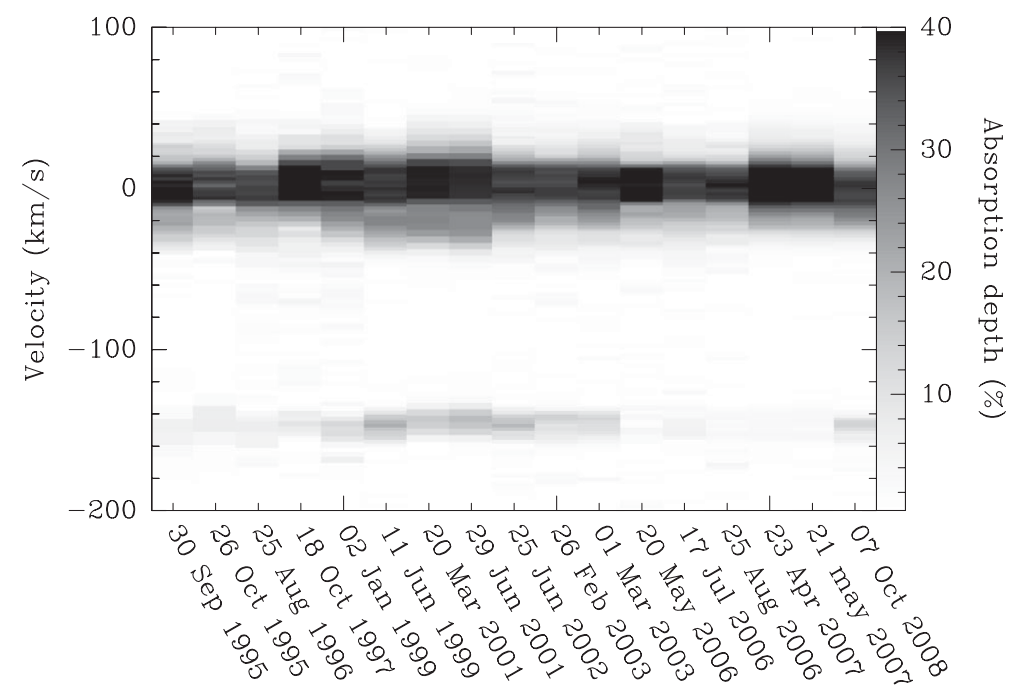

Figure 5. Evolution of the $\mathrm{HCO}^{+} 2-1$ absorption line profile observed toward PKS1830-211. Note the deepening of the $-147 \mathrm{~km} \mathrm{~s}^{-1}$ feature between 1999 and 2003, its quasi-disappearance between 2006-2007, and re-appearance in the last 2008 spectrum.

\section{Molecular absorbers as cosmological probes}

Besides allowing detailed inventory of the molecular content in distant galaxies, radio$\mathrm{mm}$ molecular absorbers are also valuable cosmological probes. The variety of molecular species provides various diagnostic tools for constraining physical properties (e.g., $\mathrm{T}_{\mathrm{CMB}}$ as a function of redshift), tracing the chemical enrichment history of the Universe (e.g., via measurements of isotopic ratios) and testing fundamental physics (e.g., the constancy of fundamental constants).

\subsection{The Cosmic Microwave Background temperature as a function of $z$}

Measuring the CMB temperature at different redshifts is important to test cosmology models: the adiabatic expansion of the Universe implies $\mathrm{T}_{\mathrm{CMB}} \propto(1+\mathrm{z})$, but models of decaying dark energy predict a different dependency: $\mathrm{T}_{\mathrm{CMB}} \propto(1+\mathrm{z})^{(1-\alpha)}$ with $\alpha>0$, (see e.g., Lima (1996), Jetzer et al. (2011)).

Observations with the COBE satellite have demonstrated that the $\mathrm{CMB}$ at $\mathrm{z}=0$ corresponds to a nearly-perfect black body and $\mathrm{T}_{\mathrm{CMB}}$ at $\mathrm{z}=0$ is measured with a very high 
accuracy: $2.72548 \pm 0.00057 \mathrm{~K}$ (Fixsen (2009)). At $\mathrm{z}>0$, two methods can be used to probe $\mathrm{T}_{\mathrm{CMB}}$ :

- multifrequency Sunyaev-Zeldovich observations toward galaxy clusters (e.g., Horellou et al. (2005), Luzzi et al. (2009));

- spectroscopic absorption study toward quasars.

Most of the measurements done so far at high redshifts have used UV spectroscopy (e.g., of CI, CII and CO, see Noterdaeme et al.(2011)). Strictly speaking, only upper limits of $\mathrm{T}_{\mathrm{CMB}}$ are derived, as the contributions from other local sources of excitation (collisions, pumping), yet largely uncertain, have to be subtracted. On the other hand, radio-mm molecular absorbers are particularly interesting, because they provide more constraints on the local excitation and physical conditions, when a variety of molecular species are detected. For example, from our ATCA $7 \mathrm{~mm}$ survey we derive rotation temperatures of $5.58 \pm 0.56 \mathrm{~K}$ and $6.23 \pm 0.41 \mathrm{~K}$ for $\mathrm{c}^{-} \mathrm{C}_{3} \mathrm{H}_{2}$ and $\mathrm{HC}_{3} \mathrm{~N}$, respectively - two species with high dipole moments (3.43 and 3.73 Debye) - while $\mathrm{T}_{\mathrm{CMB}}$ is expected to be $5.14 \mathrm{~K}$ from the standard model with adiabatic expansion. As already said, these values are upper limits of $\mathrm{T}_{\mathrm{CMB}}$, and accurate collisional rates are required for further interpretation of these rotation temperatures. We note that the two lines of sight toward the SW and NE images of PKS1830-211, presumably with different local excitation conditions, offer the possibility of a robust determination of $\mathrm{T}_{\mathrm{CMB}}$ at $\mathrm{z}=0.89$.

\subsection{Isotopic ratios}

Heavy elements (i.e. heavier than lithium) are produced in stellar interiors by the nucleosynthesis activity, and isotopic abundance ratios are good tracers of the nucleosynthesis history and thus of the chemical enrichment history of the Universe.

At $\mathrm{z}=0.89$, the look-back time is about half the age of the Universe, and the spiral galaxy toward PKS1830-211 should have an age of less than 6 Gyr. Given this timescale, stars with mass of less than $\sim 1.5 \mathrm{M}_{\odot}$ barely had the time to contribute to the enrichment of the ISM. The elemental composition of the ISM should therefore be dominated by massive stars processing.

Isotopic ratios can be derived through abundance ratios of different isotopologues of a same species. However, the interpretation of these measurements can be complicated by several effects, such as opacity of the main isotopologue, fractionation or selective photodissociation. It is therefore primordial to check the systematics by measuring the isotopologues abundance ratios in many species.

Toward PKS1830-211, we have been able to estimate the isotopic ratios of C, N, O, Si and S elements (Muller et al. $(2006,2011)$ ). The values are given in Table 3, where we also give for comparison the values of isotopic ratios in the Solar System (terrestrial) and in the carbon-rich circumstellar envelope (CSE) IRC+10216, reflecting the isotopic composition of material processed in the core of a $\sim 2 \mathrm{M}_{\odot}$ star. Apart from the ${ }^{12} \mathrm{C} /{ }^{13} \mathrm{C}$ ratio, there is a clear evolution sequence from the ratios at $\mathrm{z}=0.89$ to their terrestrial values and that in the CSE of IRC+10216. No significant differences are found between the isotopic ratios of silicon and sulfur - both processed in massive stars - in the Solar System and in IRC +10216 . In contrast, their values in the $z=0.89$ galaxy is about half lower. This may suggest an initial mass function different from that in the Milky Way.

We do not detect the deuterated variants of the $\mathrm{HCO}^{+}$or $\mathrm{HCN}$ molecules in our survey, placing an upper limit of $\mathrm{D} / \mathrm{H}<7.1 \times 10^{-4}$ and suggesting no strong deuterium fractionation enhancement.

Clearly, it would be interesting to extend these isotopic ratios measurements at various redshifts to trace the chemical enrichment history of the Universe. 
Table 3. Comparison of isotopic ratios at different states of stellar processing.

\begin{tabular}{|c|c|c|c|c|c|c|}
\hline Source & ${ }^{12} \mathrm{C} /{ }^{13} \mathrm{C}$ & ${ }^{14} \mathrm{~N} /{ }^{15} \mathrm{~N}$ & ${ }^{16} \mathrm{O} /{ }^{18} \mathrm{O}$ & ${ }^{18} \mathrm{O} /{ }^{17} \mathrm{O}$ & ${ }^{28} \mathrm{Si} /{ }^{29} \mathrm{Si}$ & ${ }^{32} \mathrm{~S} /{ }^{34} \mathrm{~S}$ \\
\hline PKS1830-211 z=0.89(SW) ${ }^{a}$ & $33 \pm 5^{\dagger}$ & $152 \pm 27$ & $69 \pm 11$ & $13_{-2}^{+3}$ & $11_{-2}^{+4}$ & $10.5 \pm 0.6$ \\
\hline Solar System (terrestrial) ${ }^{b}$ & 89 & 272 & 499 & 5.3 & 19.7 & 22.7 \\
\hline $\mathrm{IRC}+10216^{c}$ & $45 \pm 3$ & $>4400$ & $1260_{-240}^{+315}$ & $0.7 \pm 0.2$ & $15.4 \pm 1.1^{\dagger}$ & $21.8 \pm 2.6$ \\
\hline
\end{tabular}

Notes: ${ }^{\dagger}$ likely underestimated due to opacity effects.

References: (a) Muller et al. (2011); (b) Lodders (2003); (c) Cernicharo et al. (2000).

\subsection{Constancy of fundamental constants}

A cosmological variation of the value of a fundamental constant such as the proton-toelectron mass ratio $(\mu)$, would induce a shift in frequency (different from the Doppler shift) between a spectral line observed at $\mathrm{z}=0$ (e.g., in the lab) and at high-redshift. A recent and thorough review of the field is given by Uzan (2011). We focus here on $\mu$ and constraints on its variation set by molecular transitions. If all the lines had the same dependency on $\mu$, it would be impossible to distinguish a variation of $\mu$ from a Doppler shift. However, certain molecular transitions have different sensitivity to changes in $\mu$. This is the case for inversion lines of ammonia (Flambaum \& Kozlov (2007)) and some lines of methanol (Jansen et al. (2011)) with respect to pure rotational lines. This has pushed several groups to use the only two redshifted ammonia absorbers currently known, those toward B0218+357 and PKS1830-211 (Henkel et al.(2005, 2008)), to constrain variations of $\mu$ by comparing the velocity offsets between inversion lines of ammonia and rotational lines of other species (see Table 4 ).

Among the systematics that could affect the measurement, the kinematical bias (which species to take as velocity reference ?) is a severe limitation, as is proven in Figure 6, where we compare the velocity centroids of all species detected in our survey toward PKS1830-211. Several species have significant velocity offsets with respect to the bulk of other species, in particular $\mathrm{CH}_{3} \mathrm{OH}$ and $\mathrm{CH}_{3} \mathrm{NH}_{2}$ (as well as $\mathrm{NH}_{3}$, Henkel et al. (2009)), which have to be further investigated at better velocity resolution/sensitivity.

Interestingly, different lines of methanol have different sensitivity to changes in $\mu$, which could minimize the kinematical bias by removing the need for other species as velocity reference. The best case would be provided by methanol transitions with very different sensitivity to changes in $\mu$, but at similar frequencies to limit continuum morphology changes (and potential different lines of sight), and similar excitation.

Alternatively, a statistical approach to address the systematics would have to wait for the discovery of a large number of new millimeter molecular absorbers.

Table 4. Constraints on the variation of the proton-to-electron mass ratio toward redshifted mm molecular absorbers.

\begin{tabular}{|c|c|c|c|}
\hline Method & Target & $\Delta \mu / \mu\left(10^{-6}\right)$ & Ref. \\
\hline Inversion lines of $\mathrm{NH}$ & B02 & $0.74 \pm 0.47$ & $(1)$ \\
\hline Inversion lines of $\mathrm{NH}_{3}$ vs $\mathrm{HC}_{3} \mathrm{~N}$ & PKS & 0. & $(2)$ \\
\hline Inversion lines of $\mathrm{NH}_{3}$ vs $\left(\mathrm{CS}, \mathrm{H}_{2} \mathrm{CO}\right)$ & B0218+357 & $-0.35 \pm 0.12$ & (3) \\
\hline Inversion lines of $\mathrm{NH}_{3}$ vs (average of 22 species) & PKS1830-211 & $-2.04 \pm 0.74$ & (4) \\
\hline $\mathrm{CH}_{3} \mathrm{OH}$ vs (average of 22 species) & PKS1830-211 & $-1.95 \pm 0.47$ & (4) \\
\hline
\end{tabular}

References: (1) Murphy et al. (2008); (2) Henkel et al. (2009); (3) Kanekar (2011); (4) Muller et al. (2011). 


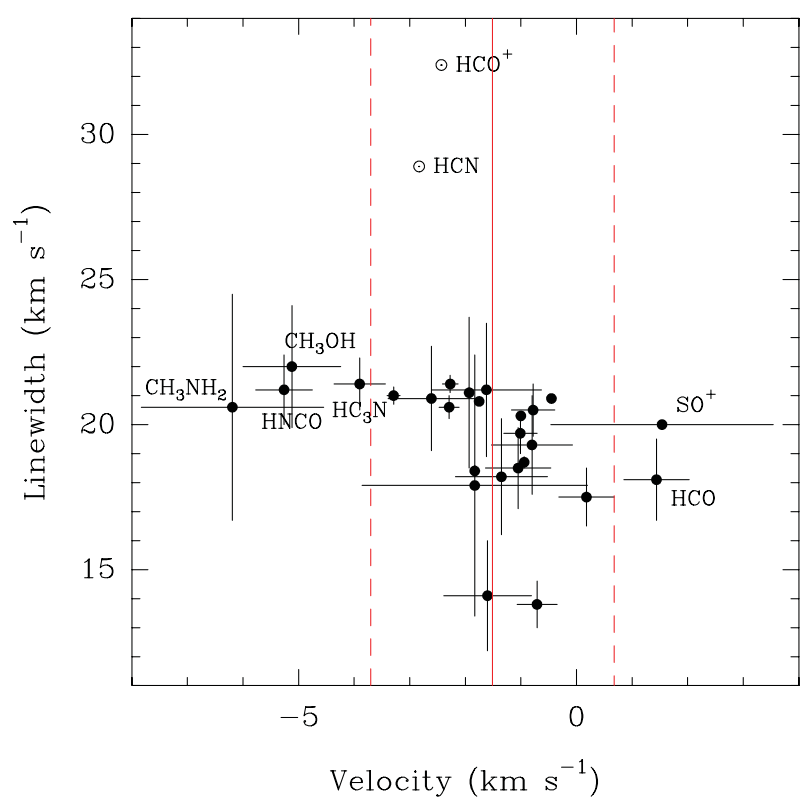

Figure 6. Diagram of the FWHM versus velocity centroids, derived from Gaussian fits of the lines toward PKS1830-211(SW). The average velocity (full line) and $3 \sigma$ dispersion (dashed lines), calculated from 22 species, are indicated. Note that $\mathrm{CH}_{3} \mathrm{NH}_{2}, \mathrm{HNCO}, \mathrm{CH}_{3} \mathrm{OH}, \mathrm{HC}_{3} \mathrm{~N}, \mathrm{HCO}$ and $\mathrm{SO}^{+}$have velocity centroids significantly different from the bulk of other species. The lines of $\mathrm{HCO}^{+}$and $\mathrm{HCN}$ are saturated.

\section{Summary and perspectives}

Redsifted millimeter molecular absorbers are currently the only objects allowing us to study the chemistry evolution at intermediate redhifts. The first unbiased spectral survey toward such a molecular absorber, that at $\mathrm{z}=0.89$ toward PKS1830-211, has yielded the (somehow surprising) detection of a large number of molecules, which can be used as powerful diagnostic tools to investigate the property of the gas in a galaxy at a look-back time of half the age of the Universe.

With the sensitivity of the ALMA and EVLA arrays, it is now possible to conduct large and deep radio/(sub)mm spectral scans: especially, redshifted molecular absorbers offer the possibility to search from the ground for molecules which could not be observed at $\mathrm{z}=0$ due to atmospheric attenuation, e.g., such as light hydrides. The sensitivity improvement increases the number of targetable continuum sources, hence chance of discovering new molecular absorption systems.

\section{Acknowledgement}

I am grateful to the organizers for inviting me to present this work and acknowledge the help and support of my collaborators.

\section{References}

Bottinelli, S., Hughes, A. M., van Dishoeck, E. F., et al., 2009, ApJ, 690, 130

Cernicharo, J., Guélin, M., \& Kahane, C., 2000, A\&A Supp., 142, 181

Chengalur, J. N., de Bruyn, A. G., \& Narasimha, D., 1999, A\&A, 343, 79

Combes, F. \& Wiklind, T., 1997, ApJ, 486, 79

Combes, F., 2008, Ap\&SSS, 313, 321 
Costagliola, F., Aalto, S., Rodriguez, M. I., et al., 2011, A\& $A$, 528, 30

Curran, S. J., Murphy, M. T., Pihlström, Y. M., et al., 2004, MNRAS, 352, 563

Curran, S. J., Whiting, M. T., Murphy, M. T., et al., 2006, MNRAS, 371, 431

Curran, S. J., Whiting, M. T., Wiklind, T., et al., 2008, MNRAS, 391, 765

Drinkwater, M. J., Combes, F., \& Wiklind, T., 1996, A\&A, 312, 771

Eckart, A., Cameron, M., Genzel, R., et al., 1990, ApJ, 365, 522

Espada, D., Peck, A. B., Matsushita, S. et al., 2010, ApJ, 720, 666

Evans, A. S., Sanders, D. B., Surace, J. A., \& Mazzarella, J. M., 1999, ApJ, 511, 730

Falco, E. E., Impey, C. D., Kochanek, C. S., et al., 1999, ApJ, 523, 617

Fixsen D. J., 2009, ApJ, 707, 916

Flambaum, V. V. \& Kozlov, M. G., 2007, Phys. Rev. Lett., 99, 801

García-Burillo, S., Combes, F., Neri, R., et al., 2007, A\& A, 468, L71

Gardner, F. F. \& Whiteoak, J. B. ,MNRAS, 175, 9

Gérin, M., Phillips, T. G., Benford, D. J., et al., 1997, ApJ 488, 31

Gérin, M., de Luca, M., Goicoechea, J. R., et al., 2010, A\& A, 521, L16

Henkel, C., Jacq, T., Mauersberger, R., Menten, K. M., \& Steppe, H., 1987, A\&\&A, 188, L1

Henkel, C., Mauersberger, R., Wiklind, T., et al., 1993, A\&A, 268, L17

Henkel, C.; Jethava, N.; Kraus, A., et al., 2005, A\&SA, 440, 893

Henkel, C., Braatz, J. A., Menten, K. M., \& Ott, J., 2008, A\&A, 485, 451

Henkel, C., Menten, K. M., Murphy, M. T., et al., 2009, A\&A A, 500, 725

Horellou C., Nord M., Johansson D., \& Lévy A., 2005, A\& $A, 441,435$

Israel, F. P., van Dishoeck, E. F., Baas, F., et al., 1990, A\&A A, 227, 342

Jauncey, D. L., Reynolds, J. E., Tzioumis, A. K., et al., 1991, Nature, 352, 132

Jansen, P., Xu, L.-H., Kleiner, I., Ubachs, W., \& Bethlem, H. L., 2011, Phys. Rev. Lett., 106, 801

Jetzer P., Puy D., Signore M., \& Tortora C., 2011, General Relativity and Gravitation, 43, 1083

Jin, C., Garrett, M. A., Nair, S., et al., 2003, MNRAS, 340, 1309

Kanekar, N., Carilli, C. L., Langston, G. I., et al., 2005, Phys. Rev. Lett., 95, 1301

Kanekar, N., 2011, ApЛ, 728, L12

Lima J. A. S., 1996, Physical Review D, 54, 2571

Liszt, H. \& Lucas, R., 2004, A\&A, 428, 445

Liszt, H. S., Pety, J., \& Lucas, R., 2008, A\&A, 486, 493

Lodders, K., 2003, ApJ, 591, 1220

Lucas, R. \& Liszt, H., $1998, A \& A, 337,246$

Lucas, R. \& Liszt, H. S., 2000, A\& $A$, 358, 1069

Luzzi G., Shimon M., Lamagna L., et al., 2009, ApJ, 705, 1122

Martín, S., Mauersberger, R., Martín-Pintado, J., Henkel, C., \& García-Burillo, S., 2006, ApJS, 164,450

Martín, S., Krips, M., Martín-Pintado, J., et al., 2011, A\&3A, 527, 36

Mathur, S. \& Nair, S., 1997, ApJ, 484, 140

Menten, Karl M. \& Reid, Mark J., 1996, ApJ, 465, L99

Menten, K. M., Carilli, C. L., \& Reid, M. J., 1999, ASP Conf. Ser., 156, 218

Menten, K. M., Güsten, R., Leurini, S., et al., 2008, A\&A A, 492, 725

Müller, H. S. P., Thorwirth, S., Roth, D. A., \& Winnewisser, G., 2001, A\&AA, 370, 49

Muller, S., Guélin, M., Dumke, M., et al., 2006, A\&A, 458, 417

Muller, S., Guélin, M., Combes, F., \& Wiklind, T., 2007, A\&A, 468, L53

Muller, S. \& Guélin, M., 2008, A\&AA, 491, 739

Muller, S. \& Dinh-V-Trung, 2009, ApJ, 696, 176

Muller, S., Beelen, A., Guélin, M., et al., 2011, A\&SA, in press, [arXiv:1104.3361]

Murphy, M. T., Curran, S. J., \& Webb, J. K., 2003, MNRAS, 342, 830

Murphy, M. T., Flambaum, Victor V., Muller, S., \& Henkel, C., 2008, Science, 320, 1611

Nair, S., Jin, C., \& Garrett, M. A., 2005, MNRAS, 362, 1157

Noterdaeme, P., Petitjean, P., Srianand, R., et al., 2011, A\&A, 526, L7

Nummelin, A., Bergman, P., Hjalmarson, A., et al., 2000, ApJ Supp., 128, 213 
Ohishi, M., Irvine, W. M., \& Kaifu, N., 1992, IAU Symp. 150, 171

Omar, A., Anantharamaiah, K. R., Rupen, M., \& Rigby, J., 2002, A\&A, 381, L290

Patnaik, A. R., Browne, I. W. A., King, L. J., et al., 1993, MNRAS, 261, 435

Pickett, H. M., Poynter, R. L., Cohen, E. A., et al., 1998, J. Quant. Spectrosc. \& Rad. Transfer, 60,883

Seaquist, E. R. \& Bell, M. B., 1986, ApJ, 303, L67

Seaquist, E. R. \& Bell, M. B., 1990, ApJ, 364, 94

Turner, B. E., 2000, ApJ, 542, 837

Uzan, J.-P., 2011, Living Reviews in Relativity, 14, 2

Wang, M., Henkel, C., Chin, Y.-N., et al., 2004, A $\mathcal{E A}$, 422, 883

Wang, M., Chin, Y.-N., Henkel, C., Whiteoak, J. B., \& Cunningham, M., 2009, ApJ, 690, 580

Wiklind, T. \& Combes F. 1995, A\&A, 299, 382

Wiklind, T. \& Combes F. 1996a, A\& A, 315, 86

Wiklind T. \& Combes F. 1996b, Nature, 379, 139

Wiklind T. \& Combes F. 1997a, A\&A, 324, 51

Wiklind T. \& Combes F. 1997b, A\&A A, 328, 48

Wiklind T. \& Combes F. 1998, ApJ, 500, 129

Wiklind, T. \& Combes, F., 1999, arXiv:astro-ph/9909314

Winn, J. N., Kochanek, C. S., McLeod, B. A., et al., 2002, ApJ, 575, 103

\section{Discussion}

KarL Menten: In your plot of derived $\mathrm{T}_{\mathrm{CmB}}$ values, two species have about $3 \mathrm{~K}$ with apparently small error bars. What is the explanation of this?

S. Muller: Those two points correspond to the rotation temperatures derived for $\mathrm{H}_{2} \mathrm{CCO}$ and $\mathrm{H}_{2} \mathrm{CCN}$. Both are detected with limited signal-to-noise ratios, and $\mathrm{H}_{2} \mathrm{CCN}$ has a myriad of hyperfine components. We are investigating if those points and their error bars were not underestimated.

RAiner Mauersberger: Two of the strongest local central sources of galaxies, Sgr A and Cen A, show absorption. Why does this encouraging statistics not extend to more distant sources?

S. MulLER: This is likely due to the combination of small number of bright radio$\mathrm{mm}$ sources at high-z and clumpiness of the molecular gas, resulting in a rare chance of alignment. In addition, there is a selection bias against background sources with high extinction, for which the redshift of an intervening object could not be known from optical spectroscopy. This might change soon with the increase in instantaneous bandwidth and sensitivity of the new generation of telescopes at $\mathrm{mm} / \mathrm{cm}$ wavelengths.

MicheL GUÉLIN: I would like to stress that molecular line absorption measurements yield more reliable molecular abundance ratios than emission line measurements, especially in medium and low density gas, as the excitation temperature is close to the cosmic background temperature. This is important for the derivation of isotopic abundance ratios. 